\title{
The effect of appropriate and inappropriate stimulus color on odor discrimination
}

\author{
Richard J. Stevenson and Megan OAten \\ Macquarie University, Sydney, New South Wales, Australia
}

\begin{abstract}
Color can strongly affect participants' self-report of an odor's qualities. In Experiment 1, we examined whether color influences a more objective measure of odor quality, discrimination. Odor pairs, presented in their appropriate color (e.g., strawberry and cherry in red water), an inappropriate color (e.g., strawberry and cherry in green water), or uncolored water were presented for discrimination. Participants made significantly more errors when odors were discriminated in an inappropriate color. In Experiment 2, the same design was utilized, but with an articulatory suppression task (AST), to examine whether the effect of color was mediated by identification or by a more direct effect on the percept. Here, the AST significantly improved discrimination for the inappropriate color condition, relative to Experiment 1. Although color does affect a more objective measure of odor quality, this is mediated by conceptual, rather than perceptual, means.
\end{abstract}

When an odor is smelled under laboratory conditions, this is a very different experience from when the same odor is smelled naturalistically. One key difference is that the odor's source can generally be seen. Several investigators have explored one particular feature of the visual world - namely, color - and the effect this has on odor perception. The presence of a color increases false alarm rates for odor detection (e.g., Engen, 1972) and increases judgments of orthonasal (sniffed) odor intensity, independently of the demand characteristics of the experiment (e.g., Zellner \& Kautz, 1990). Inappropriate color adversely affects the hedonic ratings of food (e.g., Wheatley, 1973), to which odor contributes significantly via retronasal olfaction (i.e., via the nasopharynx). Color also affects the likelihood of correctly identifying an odor or a flavor-an odor-taste combination presented orally (e.g., Davis, 1981; DuBose, Cardello, \& Maller, 1980). Relatedly, and the focus of this article, color can significantly affect the way in which participants describe their olfactory experience - that is, odor quality (e.g., DuBose et al., 1980; Kappes, Schmidt, \& Lee, 2006; Morrot, Brochet, \& Dubourdieu, 2001).

The basis of color effects on odor do not rest upon one single mechanism. Although color-mediated odor intensity effects appear to result from just the presence of any color (Zellner \& Whitten, 1999), the basis for hedonic and qualitative effects may depend on the color's impact on odor identification. In this case, the appropriateness of the color for a particular odor will affect the likelihood of successful identification (Blackwell, 1995). Indeed, appropriately coloring an odor improves identification, and successful identification appears to increase participants' positive hedonic evaluation of an odor (Zellner, Bartoli,
\& Eckard, 1991). If color then aids either correct (if it is an appropriate color) or incorrect (if inappropriate) identification, this could account for why participants describe miscolored odors (or flavors) as smelling like something else - typically, something more normally associated with that color. Zellner et al. (1991) have suggested that color may activate a subset of verbal labels based on preexisting knowledge of color-odor associations, which then form a possible pool of names from which participants may select. Odor identification is typically hard when no external cues are present (e.g., Cain, 1979), and significant errors in identification have been documented (e.g., Cain \& Potts, 1996). Thus, color-aided identification, even when incorrect, could provide the basis for participants' description of an odor, even when that description is considerably at odds with the actual identity of the stimulus. However, participants' self-reports of odor quality when the target odor is presented in an inappropriate color are so different from their reports when the odor is appropriately colored that it raises the question of whether a purely semantic explanation can suffice. To illustrate this point, we will describe below three studies in which such effects have been observed.

DuBose et al. (1980) explored participants' qualitative judgments of the flavor of lime, orange, cherry, and water, under conditions of appropriate and inappropriate coloring. For cherry, for example, whereas $70 \%$ of the participants regarded this as having a cherry-like flavor when red, only $33 \%$ did so when it was colored green. In fact, nearly $41 \%$ of the sample believed green-cherry to be citrus (lemon/lime), whereas no participant regarded the red-colored cherry flavor as being citrus-like. A similar, if not more dramatic, finding was obtained by Morrot et al.

R. J. Stevenson, richard.stevenson@psy.mq.edu.au 
(2001). Here, untrained participants evaluated the flavor profile (i.e., provided a verbal description) of white wine, white wine colored red, and red wine. The participants described the red-colored white wine as having a flavor profile almost identical to that of red wine, although under blind conditions, the red-colored white wine and the white wine were indiscriminable. Finally, Kappes et al. (2006) reported not only that color influenced the flavor profile that participants gave to uncolored versus appropriately colored carbonated soft drinks, but also that it affected the participants' evaluation of somatosensory and taste qualities too. Two points are worth noting about these three studies. First, all involved retronasal olfaction; that is, the participants did not sniff the stimuli but, rather, perceived the odors via the nasopharynx. Retronasal perception has been reported to result in poorer odor identification (e.g., Pierce \& Halpern, 1996), which, if a semantic account is correct (i.e., one based on verbal identification), might exaggerate the effect of color on odor quality perception. Second, all of these studies utilized self-report, rather than an objective measure of discrimination. One might expect self-report measures to be more vulnerable to the effects of verbal mediation, because the task itself is overtly verbal (i.e., a description of sensory experience).

An alternative possibility is that color cues may automatically activate olfactory representations that have previously been associated with that color. This could be conceptualized at a generic level (association of certain colors with, say, fruit-like odors, thus generating a fruit-like percept) or more specific (where the stochastic nature of activating a particular representation results in one of a subset of likely candidates becoming activated). Several findings suggest that this possibility should be given serious consideration. First, odor names, in the absence of olfactory stimulation, activate what are believed to be primarily perceptual processing areas of the brain that are associated with olfaction (González et al., 2006), and appropriate color-odor pairs activate the secondary olfactory cortex (Osterbauer et al., 2005). Second, not only did Zellner et al. (1991) suggest that colors may activate a pool of purely semantic (verbal) labels, they also suggested that colors might activate a similar set of perceptual representations. Third, odors appear to have quite consistent and nonidiosyncratic associations with color, suggesting a much stronger association than one based purely on past experience (Gilbert, Martin, \& Kemp, 1996). Fourth, Cain and Potts (1996) found that when participants misidentified an odor and were later given a surprise recognition memory test, they would often indicate that the misidentified odor (which they had switched) had actually occurred during the earlier part of the experiment. That is, if a participant misidentified lemon as vinegar and then vinegar was included in the recognition memory test, he or she tended to indicate that such an odor had been experienced earlier in the experiment. Taken together with the apparently striking nature of the misdescriptions that can occur when an odor is inappropriately colored, this suggests the possibility that color may lead to a perceptual change in what the person experiences and, thus, reports. In this case, seeing a colored fluid may activate a representation, which may then override that produced by bottom-up odor-driven processing or at least place limits on the final representation, which is formed.

There are three related differences between a primarily semantic and a primarily perceptual account of how color may affect odor quality. First, according to the perceptual account, color directly affects the percept, whereas for the semantic account, it affects identification and, hence, access to semantic knowledge about the odor. The second is that overt identification of the odor is unnecessary for the perceptual account but necessary for the semantic account. The third is that the appropriateness of the color in which the odor is embedded should have different effects on a discrimination task, dependent on whether color exerts a primarily semantic or perceptual effect on odor perception. In the experiments reported below, we examined these predictions.

\section{EXPERIMENT 1}

In Experiment 1, we set out to determine whether color would affect the discriminability of three pairs of odorants-lemon and grapefruit, cut grass and mint, and cherry and strawberry - using a triangle or oddity test of discrimination. These pairs were selected because they share a common associated color (yellow, green, and red, respectively), and each pair was presented twice in three within-participants conditions: first, in the color appropriate for that pair (e.g., discrimination of lemon and grapefruit presented in yellow food coloring); second, in a color inappropriate for that pair (e.g., discrimination of cut grass and mint in red food coloring); and third, in plain uncolored water (e.g., discrimination of cherry and strawberry dissolved in plain water). On the basis of the two accounts described in the introduction, two different patterns of outcomes might be predicted, relative to the baseline uncolored condition. Where color directly activates an odor representation, this should result in poorer discrimination in both the appropriate and the inappropriate color conditions, because the uniform color (e.g., red cherry vs. red cherry vs. red strawberry or green cherry vs. green cherry vs. green strawberry) will be more likely to generate a similar percept, making discrimination harder on the average. Where color assists identification, this should improve discrimination in the color-appropriate condition but worsen it in the color-inappropriate condition. In this case, an appropriate color will facilitate identification and, thus, assist discrimination as naming is known to do (e.g., Rabin, 1988). For the inappropriate color, this is likely to facilitate incorrect identification, leading to poorer discriminative performance.

Prior to the experiment, we were concerned that the participants might ignore the color of the stimuli, since the instructions asked the participants to focus on discriminating their smell. Although this would not be expected to affect our prediction derived from the perceptual account (i.e., an automatic effect), it certainly might affect whether color was used as a cue to identification (i.e., a controlled effect). Consequently, the participants were randomly assigned either to an incidental condition, in 
which no mention of color was made, or to an alerting condition, in which they were asked to tick a box if they encountered a colored stimulus. Finally, odor quality is probably the primary but not the sole determinant of odor discrimination - since differences in intensity or hedonics could be sufficient - and so, prior to Experiment 1, we piloted the stimuli to ensure that the color pairs were similar. In addition, the pilot confirmed that the pairs were appropriately matched with the colors we had selected, but to make sure, we conducted a further test of colorodor appropriateness after the discrimination phase of this experiment was complete.

\section{Method}

Participants. Thirty participants (mean age $=20.3$ years, $S D=$ 2.9) took part for course credit, of which 18 were female and 12 male. These 30 were randomly assigned to one of two conditionsincidental $(n=15)$ or alerting $(n=15)$ - by order of arrival for the experiment. No participant reported being color blind or of having an upper respiratory tract infection during testing, as with Experiment 2.

Materials. Six odorants were formed into three color-odor pairs. The members of the red odor pair were cherry (Dragoco, $1.0 \mathrm{~g} / \mathrm{L}$ ) and strawberry (Quest, $1.0 \mathrm{~g} / \mathrm{L}$ ). The members of the yellow odor pair were citral (lemon; Dragoco, $0.028 \mathrm{~g} / \mathrm{L}$ ) and grapefruit (Quest, $1.2 \mathrm{~g} / \mathrm{L}$ ). The members of the green odor pair were Cis-3-Hexanol (cut grass; Sigma, $0.15 \mathrm{~g} / \mathrm{L}$ ) and Carvone (mint; Dragoco, $0.11 \mathrm{~g} / \mathrm{L}$ ). Colored solutions were made by adding an odorless food coloring (Caroline Cake Decoration Company), at the following concentrations: red, $1 \mathrm{~g} / \mathrm{L}$; yellow, $0.2 \mathrm{~g} / \mathrm{L}$; green, $0.25 \mathrm{~g} / \mathrm{L}$. All the stimuli were presented in 50-ml aliquots in transparent plastic screw top jars, in which the color of the solution was clearly visible both prior to sniffing and after removal of the lid during sniffing. When a color was absent - that is, when the odors were just presented in water-there was no visual cue to differentiate each member of an odor pair.

Procedure. There were two components to the test session. The first involved 18 discrimination trials, using the oddity or triangle test method. On each trial, the participants received the following instructions. "You will be asked to smell three odors. Two of them will be the same and one will be different. Your job is to spot the different one - the odd one out." The experimenter then instructed the participants to remove the lid from the first container and sniff the odor three times. After the lid had been replaced, the participants waited $20 \mathrm{sec}$ before being instructed by the experimenter to repeat this process for a second odor. This interstimulus interval was deployed so as to minimize adaptation. A further $20 \mathrm{sec}$ elapsed before they were told to smell the third odor. The participants were then instructed to identify which of the three was the different one, the odd one out, by writing down the position number of that odor in their response booklet. For the participants in the incidental condition, nothing further was asked of them; however, the participants in the alerting condition were asked to make a tick in their response booklet every time they were asked to sniff a colored solution. No other instructions pertaining to color were provided. The process described above was then repeated for the remaining 17 discrimination trials. A 1-min intertrial interval separated each trial.

The 18 trials were composed of three types: 6 trials on which the odors to be discriminated were presented in their appropriate color (i.e., 2 of mint and cut grass in green, 2 of lemon and grapefruit in yellow, and 2 of strawberry and cherry in red); 6 trials on which the odors to be discriminated were presented in an inappropriate color (i.e., 1 of mint and cut grass in yellow and 1 in red, 1 of lemon and grapefruit in green and 1 in red, and 1 of strawberry and cherry in green and 1 in yellow); and 6 trials on which the odors to be discriminated were presented without any color cues (i.e., 2 of mint and cut grass in plain water, 2 of lemon and grapefruit in plain water, and 2 of strawberry and cherry in plain water). The position of the odd stimulus and whether an odor was presented once or twice within each trial were counterbalanced across participants. Presentation order of the trial types was random, with the caveat that no two trials utilizing the same odor set should follow in succession.

The second part of the experiment immediately followed the first. The participants had placed in front of them a set of three containers: one containing red fluid, one green fluid, and one yellow fluid (at the same color concentration as that described in the Materials section). The participants were then handed a container, which held one of the six target odors in colorless water. They were instructed to smell this odor and were asked which of the three colors best matched the odor, which of the three colors offered the second best match, and which color was the poorest match. They were also told that once a color match had been made, that color could not then serve in a subsequent judgment (e.g., if red was picked as the best match for cherry, it could not serve as the second best match as well). This process was then repeated for the remaining five target odors. The presentation order of the target odors was counterbalanced across participants.

Analysis. The participants' errors for each type of discrimination trial were calculated. A score of 1 was returned for each error, giving a minimum score of 0 and a maximum score of 6 for each trial type: uncolored, appropriately colored, and inappropriately colored. For the second test, we assigned the participants' best-matched color a score of 3, the second-best match a score of 2, and the poorest match a score of 1 . In this way, we could see whether, on average, a particular odor-color pair (i.e., lemon/grapefruit-yellow, cherry/ strawberry-red, cut-grass/mint-green) received a high score (indicating that we had made a match that the participants tended to agree with) or a low score (indicating that we had made a match that the participants tended to disagree with). Finally, all of these data were found suitable for parametric testing based on satisfying the assumption of normality, homogeneity of variance, and sphericity, as with Experiment 2.

\section{Results}

Discrimination errors. The participants' discrimination errors for each trial type are detailed in Table 1 by condition. These data were analyzed by a two-way ANOVA with one repeated measure, trial type (uncolored vs. appropriate vs. inappropriate), and one between factor, noticing (incidental vs. alerting). The principal result of interest was the main effect of trial type, which was significant $\left[F(2,56)=4.54, M S_{\mathrm{e}}=0.68, p<.02\right]$. As can be seen in Table 1, the participants tended to make the most errors when an odor was presented for discrimination in an inappropriate color. We tested the difference between the

Table 1

Mean Discrimination Errors (With Standard Deviations)

by Trial Type and by Whether Participants Were Instructed (Alerting) or Not (Incidental) to Notice the Color of the Stimulus, for Experiments 1 and 2

\begin{tabular}{|c|c|c|c|c|c|c|}
\hline \multirow{3}{*}{$\begin{array}{l}\text { Instruction } \\
\text { Condition }\end{array}$} & \multicolumn{6}{|c|}{ Trial Type } \\
\hline & \multicolumn{2}{|c|}{ Uncolored } & \multicolumn{2}{|c|}{$\begin{array}{c}\text { Inappropriate } \\
\text { Color } \\
\end{array}$} & \multicolumn{2}{|c|}{$\begin{array}{c}\text { Appropriate } \\
\text { Color }\end{array}$} \\
\hline & $M$ & $S D$ & $M$ & $S D$ & $M$ & $S D$ \\
\hline \multicolumn{7}{|c|}{ Experiment 1} \\
\hline Alerting & 1.3 & 1.1 & 1.9 & 1.2 & 1.2 & 1.0 \\
\hline Incidental & 0.5 & 0.7 & 1.2 & 1.1 & 0.9 & 0.7 \\
\hline Overall & 0.9 & 1.0 & 1.5 & 1.2 & 1.0 & 0.9 \\
\hline \multicolumn{7}{|c|}{ Experiment 2} \\
\hline Alerting & 1.3 & 1.1 & 1.0 & 0.8 & 0.8 & 0.8 \\
\hline Incidental & 1.3 & 1.0 & 1.2 & 1.0 & 1.3 & 0.8 \\
\hline Overall & 1.3 & 1.1 & 1.1 & 0.9 & 1.1 & 0.9 \\
\hline
\end{tabular}


trial type means, using $t$ tests with a Bonferroni-adjusted alpha (.017). There were significantly more errors in the inappropriate color condition than in the appropriate color condition $[t(29)=3.04, p<.005]$. There was a trend for more errors in the inappropriate color condition, relative to the uncolored control $[t(29)=2.43, p=.022]$, and no evidence of a difference between the appropriate color condition and the uncolored control $(t<1)$. Finally, alerting the participants to the color significantly increased the overall error rate $\left[F(1,28)=4.62, M S_{\mathrm{e}}=1.75, p<\right.$ $.05]$, but it did not affect the pattern of errors across trial types (i.e., the interaction of trial type and noticing was not significant; $F<1$ ).

We then conducted a planned contrast to determine whether the pattern of errors for each of the three odorcolor pairs differed across the factor of trial type. The same pattern was evident for each odor-color pair, with the most errors always found in the inappropriate condition and the least errors in the appropriate and uncolored control conditions. This contrast was not significant $(F<1)$.

Color-odor matches. These data were analyzed in a three-way repeated measures ANOVA, with noticing as a between factor (see above) and with odor-color pair as one within factor (red set vs. green set vs. yellow set) and target as the other (expected matching color vs. mean of expected nonmatching colors). The data from which these scores were derived are presented in Table 2, and it is evident that the color we expected to match each respective odor set corresponded with the participants' judgments too [main effect of target, $F(1,86)=203.42, M S_{\mathrm{e}}=0.21$, $p<.001]$. That is, color-odor pairs we expected to match were consistently accorded higher scores than were colorodor pairs we did not expect to match. There were no other significant effects.

\section{Discussion}

The participants made more discriminative errors when the color was inappropriate for the odor pair being discriminated. In the introduction to this experiment, we sug-

Table 2

Matching Score (Maximum = 3, Minimum = 1) for Each Odor-Color Pair for the Target Color and for the Nontarget Colors (With Standard Deviations), for Experiments 1 and 2

\begin{tabular}{|c|c|c|c|c|c|c|}
\hline \multirow[b]{2}{*}{ Stimulus } & \multicolumn{2}{|c|}{ Target Color } & \multicolumn{4}{|c|}{ Nontarget Colors } \\
\hline & $M$ & $S D$ & $M$ & $S D$ & $M$ & $S D$ \\
\hline \multicolumn{7}{|c|}{ Experiment 1} \\
\hline Strawberry & R 2.7 & 0.5 & G 1.3 & 0.5 & Y 2.0 & 0.7 \\
\hline Cherry & R 2.8 & 0.6 & G 1.4 & 0.5 & Y 1.8 & 0.6 \\
\hline Mint & G 2.8 & 0.4 & R 1.3 & 0.5 & Y 1.8 & 0.6 \\
\hline Cut grass & G 2.6 & 0.7 & R 1.4 & 0.7 & Y 2.1 & 0.6 \\
\hline Grapefruit & Y 2.5 & 0.6 & R 1.7 & 0.7 & G 1.8 & 0.8 \\
\hline Lemon & Y 2.5 & 0.7 & R 1.4 & 0.6 & G 2.0 & 0.7 \\
\hline \multicolumn{7}{|c|}{ Experiment 2} \\
\hline Strawberry & R 2.6 & 0.7 & G 1.6 & 0.7 & Y 1.8 & 0.6 \\
\hline Cherry & R 2.5 & 0.7 & G 1.5 & 0.7 & Y 1.9 & 0.7 \\
\hline Mint & G 2.9 & 0.4 & R 1.2 & 0.4 & Y 1.9 & 0.5 \\
\hline Cut grass & G 2.4 & 0.8 & R 1.5 & 0.6 & Y 2.0 & 0.6 \\
\hline Grapefruit & Y 2.7 & 0.6 & R 1.5 & 0.6 & G 1.8 & 0.6 \\
\hline Lemon & Y 2.5 & 0.6 & R 1.5 & 0.8 & G 1.9 & 0.6 \\
\hline
\end{tabular}

Note-G, green; R, red; Y, yellow. gested two possible outcomes, which were seen as being indicative of errors based on identification or perception. If color effects were based on identification, we predicted that performance would be poorest in the inappropriate color condition and best in the appropriate color condition, relative to the uncolored control. If color effects were based on perception, we predicted equally poor performance in both the appropriate and the inappropriate conditions, relative to the uncolored control.

The results here appear to fit more closely with the identification account, although we did not observe facilitated performance in the appropriate color condition, relative to the uncolored control, which may have been a result of a floor effect. That is, the discrimination testing procedure was more sensitive to errors and, thus, less sensitive to a reduction in errors - hence, a floor effect. However, what we did observe was much poorer performance in the inappropriate color condition, which was significantly worse than that in the appropriate color condition, consistent with the prediction that it is identification that underpins these effects. One way to tell more definitively whether identification is at the heart of this effect is to employ a task that prevents or retards identification during the discrimination task. If the observed effect does not persist under such conditions (i.e., poorer performance in the inappropriate color condition), we might then conclude that misidentification was responsible for the higher error rate in the inappropriate color condition here. In Experiment 2, we set out to test this.

\section{EXPERIMENT 2}

To retard odor identification during discrimination, we employed an articulatory suppression task (AST). This has been used before for exactly these type of situations (Reed, 2000; Stevenson, Sundqvist, \& Mahmut, 2007). Here, participants have to repeat the word "the" out loud during the interstimulus interval and, crucially, during the decision phase of the discrimination task, to minimize the use of a naming strategy. In both of the cited instances in which this task has been used before, the AST has not in fact produced any significant difference in performance relative to the non-AST condition, suggesting that in these cases at least, the tasks were not verbally mediated. In this experiment, we would expect that relative to Experiment 1, the AST should in fact improve discriminative performance in the inappropriately colored condition, if indeed this is mediated by semantically driven processes.

\section{Method}

Participants. Forty-eight participants completed the experiment for course credit or a small cash payment, but data from 2 were eliminated since they had completed a similar experiment before. Of the remaining 46 (mean age $=26.1$ years, $S D=10.3$ ), 30 were female and 16 male. The participants were randomly assigned to one of two conditions-incidental $(n=22)$ or alerting $(n=24)$ - by order of arrival for the experiment.

Materials. The materials were identical to those in Experiment 1.

Procedure. The basic procedure was similar to that in Experiment 1 , except for the following. Prior to discrimination testing, the participants took part in three training trials to ensure that they grasped the nature of the task. The first training trial simply took the 
participants through the basic discrimination procedure, in the same manner as that described for Experiment 1. In the second practice trial, the participants were asked to sniff a target odor, and the moment they had completed sniffing, they were immediately asked to say "the, the ..." aloud until told to stop by the experimenter. They were told to stop just before they sniffed the second stimulus. Again, the moment sniffing was complete, the AST was commenced again until just before they sniffed on the third trial. After sampling the third odor, the participants started the AST again and kept at it until they had noted down the number of the odd trial. This was carefully policed by the experimenter. Interstimulus and intertrial intervals were the same as those in Experiment 1. For the third practice trial, the participants in the incidental condition repeated the same AST and discrimination procedure as those described above. However, the participants in the alerting condition were asked to engage in the AST and discrimination procedure as well as indicating whether or not each solution was colored, in the same manner as that described for Experiment 1. This was then followed by the 18 test discrimination trials, which were made up of the same trial types as those in Experiment 1. Finally, the participants completed the color-odor matching task in the same way as that described for Experiment 1

\section{Results}

Discrimination errors. Table 1 details discrimination errors for each trial type by whether or not the participants were asked to notice the color. The same ANOVA design as that in Experiment 1 revealed no significant main effects of trial type or of noticing and no interaction between these variables (all $F \mathrm{~s}<1$ ). Thus, it appears that the use of the AST may eliminate or retard the effect of inappropriate color on discriminative performance that we observed in Experiment 1.

To ascertain this more directly, we compared performance across these two experiments, since they were procedurally identical (excepting, of course, the AST in Experiment 2) and drew on a demographically similar pool of participants. To compare them, we used a three-way ANOVA, with trial type as a repeated measure (uncolored vs. appropriately colored vs. inappropriately colored) and experiment (no AST vs. AST) and noticing (incidental vs. alerting) as between factors. The analysis revealed a significant interaction between trial type and experiment $\left[F(2,144)=3.16, M S_{\mathrm{e}}=0.82, p<.05\right]$. To explore this further, we examined a specific contrast - namely, the mean of the inappropriate color condition minus the mean of the uncolored and appropriately colored conditions combined. We then compared this difference score between experiments, using an independent $t$ test. With alpha set at .017, the outcome of these two experiments significantly differed on this contrast $[t(74)=2.44, p<$ $.017]$, indicating that the effect observed in Experiment 1 in the inappropriate color condition was significantly attenuated by the inclusion of the AST in Experiment 2.

The comparative ANOVA revealed no interaction of trial type and noticing $(F=1.15)$ and no main effect of experiment $(F<1)$. However, there was a significant interaction between experiment and noticing $[F(1,72)=$ $\left.8.15, M S_{\mathrm{e}}=1.20, p<.01\right]$. This suggested that whereas alerting the participants in Experiment $1(M=1.5)$ produced more errors than in the incidental condition $(M=$ $0.9)$, in Experiment 2, alerting participants $(M=1.0)$ had little effect relative to the incidental condition $(M=1.3)$.
As the single-experiment ANOVAs have already shown, there was a main effect for noticing in Experiment 1, but not in Experiment 2, which implies that the AST likely eliminated the color-alerting effect.

Color-odor matches. The same ANOVA design was utilized for these data as for those in Experiment 1 (see Table 2 for the raw data). The only significant effect was for target $\left[F(1,44)=155.50, M S_{\mathrm{e}}=0.35, p<.001\right]$, with the participants judging the target colors as significantly better matches than the nontarget colors. We were also interested in whether odor-color matching differed between Experiments 1 and 2, but when the ANOVA here was repeated with experiment added as a between factor, again only the target factor was significant $[F(1,72)=$ $\left.319.84, M S_{\mathrm{e}}=0.30, p<.001\right]$. Thus, the participants in both Experiments 1 and 2 tended to perceive the odorcolor matches in a similar manner.

\section{Discussion}

The inclusion of the AST actually improved discriminative performance in the inappropriate color condition, relative to Experiment 1. In addition, the AST interfered with the effect of getting the participants to notice the color cue, relative to Experiment 1, suggesting, overall, that the color cues exert an effect on controlled processing-identification. Thus, when the effects of noticing the color are interfered with by the AST, error rates are reduced accordingly.

\section{GENERAL DISCUSSION}

This article presents the first evidence that odor discrimination may be affected by stimulus color, a finding consistent with other reports of color's influencing participants' self-report of odor qualities when the odor stimulus is kept constant but color is manipulated (DuBose et al., 1980; Kappes et al., 2006; Morrot et al., 2001). More important, the findings of both Experiments 1 and 2 suggest that this effect is mediated by odor identification, consistent with findings reported previously that have indicated a role for identification in color-odor effects (e.g., Zellner et al., 1991). In Experiment 1, only the inappropriate color condition demonstrated a higher error rate, consistent with color's leading to misidentification of the to-be-discriminated odors, with a consequent negative impact on discriminative performance. This disruptive process might be likened to verbal overshadowing, which has been demonstrated with flavor stimuli before (Melcher \& Schooler, 1996). If color had automatically cued an associated odor percept, we would have expected poorer performance in both the appropriate and the inappropriate color conditions, and this was not observed. In Experiment 2, we attempted to disrupt identification by using an articulatory suppression task during discrimination. When this task was used, the participants no longer made more errors in the inappropriate color condition and, when contrasted to Experiment 1, made significantly fewer errors in comparison. Together, this implies that color effects here are driven by identification, rather than by color's exerting a more direct effect on the odor percept. 
The effects observed in the experiment may, of course, be peculiar to the conditions and odors employed here. First, the interstimulus interval may have encouraged naming (as a mnemonic aid), and if the interstimulus interval was removed, it is plausible that no effect of color on odor discrimination would have been observed (i.e., akin to Experiment 2). If this were the case, the conclusion one would draw would, in fact, be remarkably similar to the inference drawn from both experiments here: that no perceptual change appears to accompany the experience of colored odors; rather, that these effects manifest either when participants have the opportunity to name the odors (as we presume occurred in Experiment 1) or where identification is implicitly demanded by requests to describe the olfactory percept. A second consideration is whether the effects here are peculiar to familiar odors. In fact, color may exert a more profound effect on unfamiliar odors, since unfamiliar odors are harder to discriminate from each other (Rabin, 1988) and are described in vaguer terms than are familiar odors (Mingo \& Stevenson, 2007). Thus, the greater perceptual plasticity of unfamiliar odors may leave them open to much greater misidentification, based on incorrect color-cued naming. A similar argument could also be mounted for retronasal presentation (i.e., as a flavor) as was noted in the introduction.

Although color-odor associations may be strong and occur automatically (e.g., Dematte, Sanabria, \& Spence, 2006), this article suggests that their effect on odor quality perception may be initially delimited to misidentification, rather than being a direct effect on the percept. A key question then is whether following misidentification, the percept itself is altered in line with the misidentification. In the introduction, we reviewed several lines of evidence favorable to colors' exerting a perceptual effect on odor perception. Additional evidence comes from the finding that odor names themselves have an impact upon the hedonic ratings that participants report for the same stimulus (Herz \& von Clef, 2001). Under conditions in which an odor is given a more favorable label (i.e., parmesan cheese) or a less favorable one (i.e., vomit), evaluations change accordingly. Although verbal effects alone might offer an alternative account of this finding, they have been interpreted as indicative of perceptually based change driven by misidentification.

Although our findings may be consistent with misidentification's affecting the odor percept, a further source of evidence, which is much more extensive, is the odor imagery literature. In the simplest studies, participants are asked to form an image of an odor when given its name (e.g., imagine the smell of a lemon). This can be considered in a similar light to the effect of misidentification, where the misnamed odor is present along with its subvocally generated name. If participants can readily form an odor image, given an odor's name, it would not be too surprising to learn that the presence of a name, even an incorrect one, could then affect perception of an odor that was actually physically present. However, participants typically report great difficulty in forming odor images in response to a name (e.g., Lawless, 1997; Lindauer, 1969;
Sheehan, 1967). More generally, although some capacity for odor imagery clearly exists (Stevenson \& Case, 2005), it is both less effective and more unreliable than that observed for the other senses (e.g., Herz, 2000). On these grounds, we might suggest that misidentification is not likely to exert a strong effect on the percept. However, what it clearly can do is to provide access to a wealth of semantic information about the (misnamed) odor, which could then inform many types of olfactory judgments.

In conclusion, coloring of an odor stimulus can adversely affect discrimination, consistent with the many other findings reported in the literature of color's impacting on naming, hedonics, intensity, and quality judgments. However, the experiments reported here suggest that these effects are not exerted directly on the percept but, rather, arise primarily from misidentification. Although these results may, strictly speaking, be limited to the special circumstances of these experiments, they suggest that color effects may manifest primarily by conceptual means.

\section{AUTHOR NOTE}

The authors thank Sarah Jacek and Margery Aylet for assistance with these experiments and the Australian Research Council for their financial support. Correspondence concerning this article should be addressed to R. J. Stevenson, Department of Psychology, Macquarie University, NSW 2109, Australia (e-mail: richard.stevenson@psy.mq.edu.au).

\section{REFERENCES}

BlACKWELL, L. (1995). Visual cues and their effects on odour assessment. Nutrition \& Food Science, 5, 24-28.

CAIN, W. S. (1979). To know with the nose: Keys to odor and identification. Science, 203, 468-470.

CaIn, W. S., \& PotTs, B. C. (1996). Switch and bait: Probing the discriminative basis of odor identification via recognition memory. Chemical Senses, 21, 35-44.

DAVIS, R. G. (1981). The role of nonolfactory context cues in odor identification. Perception \& Psychophysics, 30, 83-89.

Dematte, M., Sanabria, D., \& Spence, C. (2006). Cross-modal associations between odors and colors. Chemical Senses, 31, 531-538.

DuBose, C. N., Cardello, A. V., \& Maller, O. (1980). Effects of colorants and flavorants on identification, perceived flavor intensity, and hedonic quality of fruit-flavored beverage and cake. Journal of Food Science, 45, 1393-1415.

Engen, T. (1972). The effect of expectation on judgments of odor. Acta Psychologica, 36, 450-458.

Gilbert, A. N., Martin, R., \& Kemp, S. E. (1996). Cross-modal correspondence between vision and olfaction: The color of smells. American Journal of Psychology, 109, 335-351.

González, J., Barros-Loscertales, A., Pulvermüller, F., MeSeguer, V., Sanjuán, A., Belloch, V., \& Avila, C. (2006). Reading cinnamon activates olfactory brain regions. NeuroImage, 32, 906-912.

HERz, R. S. (2000). Verbal coding in olfactory versus nonolfactory cognition. Memory \& Cognition, 28, 957-964.

HERZ, R. S., \& VON CLEF, J. (2001). The influence of verbal labeling on the perception of odors: Evidence for olfactory illusions? Perception, 30, 381-391.

KAPPES, S. M., SchmidT, S. J., \& LeE, S. Y. (2006). Color halo/horns and halo-attribute dumping effects within descriptive analysis of carbonated beverages. Journal of Food Science, 71, S590-S595.

LAWLESS, H. T. (1997). Olfactory psychophysics. In G. K. Beauchamp \& L. Bartoshuk (Eds.), Tasting and smelling (pp. 125-175). San Diego: Academic Press.

LindAUER, M. S. (1969). Imagery and sensory modality. Perceptual \& Motor Skills, 29, 203-215.

Melcher, J. M., \& SCHOOLER, J. W. (1996). The misrembrance of wines 
past: Verbal and perceptual expertise differentially mediate verbal overshadowing of taste memory. Journal of Memory \& Language, 35, 231-245.

Mingo, S., \& Stevenson, R. J. (2007). Phenomenological differences between familiar and unfamiliar odours. Perception, 36, 931-947.

Morrot, G., Brochet, F., \& Dubourdieu, D. (2001). The color of odors. Brain \& Language, 79, 309-320.

Osterbauer, R. A., Mathews, P. M., Jenkinson, M., Beckmann, C. F. Hansen, P. C., \& Calvert, G. A. (2005). Color of scents: Chromatic stimuli modulate odor responses in the human brain. Journal of Neurophysiology, 93, 3434-3441.

Pierce, J., \& Halpern, B. P. (1996). Orthonasal and retronasal odorant identification based upon vapor phase input from common substances. Chemical Senses, 21, 529-543.

RabIN, M. D. (1988). Experience facilitates olfactory quality discrimination. Perception \& Psychophysics, 44, 532-540.

REED, P. (2000). Serial position effects in recognition memory for odors. Journal of Experimental Psychology: Learning, Memory, \& Cognition, 26, 411-422.

SheEhan, P. W. (1967). A shortened form of Betts' Questionnaire upon mental imagery. Journal of Clinical Psychology, 23, 386-389.
Stevenson, R. J., \& Case, T. I. (2005). Olfactory imagery: A review. Psychonomic Bulletin \& Review, 12, 244-264.

Stevenson, R. J., Sundevist, N., \& Mahmut, M. (2007). Age-related changes in discrimination of unfamiliar odors. Perception \& Psychophysics, 69, 185-192.

Wheatley, J. (1973). Putting color into marketing. Marketing, 67, 24-29.

Zellner, D. A., Bartoli, A. M., \& Eckard, R. (1991). Influence of color on odor identification and liking ratings. American Journal of Psychology, 104, 547-561.

Zellner, D. A., \& Kautz, M. A. (1990). Color affects perceived odor intensity. Journal of Experimental Psychology: Human Perception \& Performance, 16, 391-397.

Zellner, D. A., \& Whitten, L. A. (1999). The effect of color intensity and appropriateness on color-induced odor enhancement. American Journal of Psychology, 112, 585-604.

(Manuscript received December 21, 2006; revision accepted for publication November 19, 2007.) 